\title{
Lotka-Volterra Model of Macro-Evolution on Dynamical Networks
}

\author{
François Coppex ${ }^{1}$, Michel Droz ${ }^{1}$, and Adam Lipowski ${ }^{1,2}$ \\ 1 Institut de Physique Théorique, Université de Genève, \\ quai E. Ansermet 24, 1211 Genève 4, Switzerland, \\ 2 Faculty of Physics, A. Mickiewicz University, 61-614 Poznań, Poland
}

\begin{abstract}
We study a model of a multi-species ecosystem described by Lotka-Volterra-like equations. Interactions among species form a network whose evolution is determined by the dynamics of the model. Numerical simulations show power-law distribution of intervals between extinctions, but only for ecosystems with sufficient variability of species and with networks of connectivity above certain threshold that is very close to the percolation threshold of the network. Effects of slow environmental changes on extinction dynamics are also examined.
\end{abstract}

\section{Introduction}

Lotka-Volterra models of interacting species have a well established role in population ecology [1]. Being inspired by an oscillatory behavior in some preypredator systems, they are typically used to model populations on time scale shorter than lifetime of describing species. It means that long-term properties of ecosystems (macro-evolution) are usually not captured within such an approach. On the other hand, models used to describe macro-evolution very often use a dynamics that operates at the level of species rather than individuals. Such coarse-grained models usually refers to the notion of fitness of a species that is not commonly accepted [2]. Recently, there has been some attempts to study macro-evolution using models equipped with dynamics that operates at the level of individuals $[3,4,5]$. Taking into account that Lotka-Volterra models are relatively successful in describing many aspects of population dynamics it would be desirable to apply such an approach also to macro-evolution. Some time ago Abramson introduced a discrete version of Lotka-Volterrra ecosystem [6] and studied certain characteristics of extinctions. His model is an example of a one-dimensional food chain with $N(\sim 100)$ trophic levels and a single species occupying a given trophic level. Since in realistic food webs $N \sim 4-6$ with typically many species belonging to a given trophic level $[7,8]$, these are highly nonrealistic assumptions. Nevertheless, extinction dynamics in Abramson's model shows some features that are characteristic to Earth biosystem.

In the present paper we introduce a Lotka-Volterra model that describes a simplified ecosystem of $N$ species of predators and one species of preys. Our model can be thus considered as a simple food web model with only two trophic levels. Competition between predator species is described by a certain random 
network [9] of interactions whose evolution is coupled with dynamics of the model. Namely, when a certain species becomes extinct (i.e., its density falls below a certain threshold) it is replaced by new species with a newly created set of interactions with some of existing species. Despite obvious simplifications the model exhibits some properties that are typical to more complicated ecosystems, as for example power-law distributions of intervals between extinctions. Within our model we can also examine how robust this power-law distribution is. We find that under certain conditions, as for example very sparse interactions between species, or too strong dominance of a small group of species, these power-law characteristics disappear and the model is driven into a regime where extinctions have exponential distributions or where there are no extinctions and the ecosystem enters a steady state. In our opinion, such regimes might be relevant when a restricted (either in space or time) evolution of an ecosystem or its part is studied. Interestingly, a threshold value of connectivity that separates powerlaw extinctions and steady state is very close to the percolation threshold of the random network of inter-species interactions.

One of the important results coming from applying statistical physics models to biological evolution is that avalanches of extinctions do not require external factors to trigger them, but might be a natural consequence of the dynamics of an ecosystem. As a result, these external factors, as e.g., climate changes, solar activity or impact of a big meteorite, are very often neglected in such studies [10]. But such factors certainly affect the ecosystem and there is a good evidence of it [11]. One possibility to take external factor(s) into account in our model is to modify a growth rate of prey. modify a growth rate of prey. Since dynamics of the model is nonlinear, such a change might have more dramatic consequences than merely a change of densities of species. And indeed we noticed that dynamics of extinctions is strongly dependent on the growth rate. It turns out, that in our model abundance of preys leads to a larger frequency of extinctions, and in periods of hunger there are less extinctions. This is clearly due to nonlinearity of the dynamics. Larger growth rate increases the density of preys that in turn increases densities of predators. With increased densities, dynamics becomes more competitive and extinctions become more frequent. Such a periodically modulated growth rate leaves some traces also in the probability distribution of extinctions. It might be intersting to notice that paleontological data also show some traces of periodic events, but their proper understanding is still missing [11, $12]$

\section{The Model and Numerical Calculations}

We study a Lotka-Volterra ecosystem that consists of $N$ species of predators with densities $\rho_{i}(i=1,2, \ldots, N)$ who are all feeding on one species of preys with density $\rho_{0}$. We assume that each predator species $i$ is characterized by a parameter $k_{i}\left(0<k_{i}<1\right)$ that enters evolution equations of the model through death and growth terms

$$
\dot{\rho_{0}}=g(t) \rho_{0}\left(1-\rho_{0}\right)-\frac{\rho_{0}}{N} \sum_{i=1}^{N} f\left(k_{i}\right) \rho_{i}
$$




$$
\dot{\rho}_{i}=-\rho_{i}\left(1-\rho_{0}\right)+f\left(k_{i}\right) \rho_{i} \rho_{0}\left(1-\frac{k_{i} \rho_{i}+\sum_{j}^{\prime} k_{j} \rho_{j}}{k_{i}+\sum_{j}^{\prime} k_{j}}\right),
$$

where $i=1,2, \ldots, N$. In our model we assume that species interact mainly through environmental capacity terms (the last term in Eq. (2)). Namely, the growth rate of a given species $i$ is reduced not only due to its density but also due to weighted (with the factor $k$ ) densities of a group of randomly selected neighbor species. In Eq. (2) summation over these neighbouring species is denoted by $\left(\sum^{\prime}\right)$. Approximately, we might interpret the coefficient $k_{i}$ as the size of organisms of $i$-th species - the bigger they are the stronger their role in the environmental capacity term. We also assume that the growth rate of preys is corrected by the environmental capacity term and due to external factors might be a slowly varying function of time. In principle, external factors might affect also other terms of model (1)-(2), but for simplicity we restrict its influence only to the growth rate of preys. Functions $g(t)$ and $f(k)$ will be specified later.

Differential equations (1)-(2) are solved using Runge-Kutta fourth-order method. Each time a density of a certain species drops below a threshold value which we fix as $\varepsilon=10^{-7}$ we consider such a species as extinct [13]. Such species is then replaced by a new species with a randomly assigned density (from the interval $(0,1))$, the coefficient $k(0<k<1)$ that is randomly drawn from the distribution $p(k)$, and a new set of neighbors (all links of the 'old' species are removed). With such rules the model rather describes $N$ niches, and we assume that a time to create a species that will occupy a niche is relatively short comparing to the typical lifetime of species [14].

We assume that a newly created species makes $z$ links with randomly selected neighbors. Links are not directional so a newly created species will also enter the evolution equation of species it is neighbouring. If the extinct species would be chosen randomly the network of interactions would have been a random graph. However, it is the dynamics (1)-(2) that determines which species are extinct. Thus, extinct species are not selected randomly and the resulting network is in general not a random graph.

\section{Results}

In the following we describe numerical results obtained for some particular cases of model (1)-(2).

\subsection{Intervals between Extinctions}

Various paleontological data suggest that dynamics of extinctions has some power-law distributions of sizes or durations [11]. In our model we measured time intervals $t$ between successive extinctions. In this calculations we used a constant growth term of preys $g(t) \equiv 1$. We examined two cases: (i) model I: $f\left(k_{i}\right) \equiv 1$ and (ii) model II: $f\left(k_{i}\right)=k_{i}$. Unless specified otherwise we select $k_{i}$ randomly with a homogeneous distribution on the interval $(0,1)(p(k)=1)$. Our results are shown in Fig. 1 . In the simplest case, model I with $z=2$ and $k_{i} \equiv 1$ 
(i.e., all species during the evolution have identical $k_{i}$ ) we obtain exponentially decaying distribution of intervals between extinctions $P(t)$. Such a decay is also seen for model I $(\mathrm{z}=4)$ with linear distribution of $k_{i}$ namely $p(k)=2 k$. We expect that such a behavior appears when a distribution of $k_{i}$ in the ecosystem is relatively narrow and shifted toward unity. Such an effect might be due to the small width of distribution $p(k)$ (i.e., a distribution from which we draw $k_{i}$ ) or might be dynamically generated as in model II. In this case even though $k_{i}$ are chosen from a homogeneous distribution, the dynamics favours large $k_{i}$ species (due to their larger growth rate) and they dominate the ecosystem. When the distribution of $k_{i}$ in the ecosystem is more uniform (model I with $p(k)=1$ ) our simulations suggest that $P(t)$ decays as a power law. Let us notice, however, that a power-law behavior is seen only on approximately one decade and we cannot exclude that on a larger time scale a different (perhaps exponential) behavior appears as was already observed in some other macroevolutionary models [3]. Let us also notice that for model I with $p(k)=\frac{1}{2} k^{-1 / 2}$ the power-law distribution $P(t)$ seems to decay as $t^{-2}$, i.e., with the exponent consistent with some paleontological data [11] as well as with predictions of some other models [4].

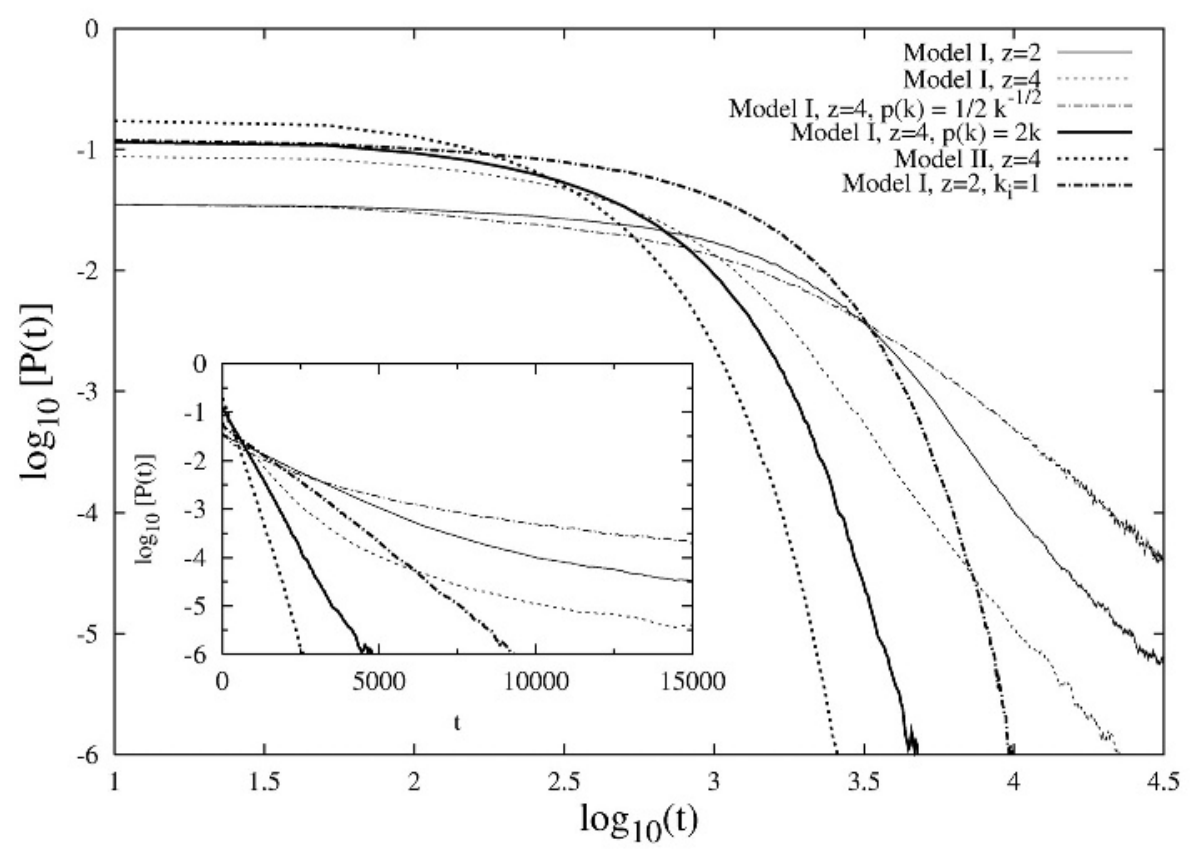

Fig. 1. Probability distribution of intervals between successive extinctions $P(t)$ calculated for some particular cases of model (1)-(2) for $N=100$. Inset shows the same data but plotted on a lin-log scale 
However, a power-law decay of $P(t)$ is seen only for sufficiently large $z$. When $z$ is too small, we observed that the ecosystem enters the steady state where all $\rho_{i}$ are positive and there are no extinctions. This is probably due to the fact that the competition among predators is too weak (or rather too sparse). To examine the transition between this two regimes in more detail we measured the averaged time between extinctions $\tau$ and the results are seen in Fig. 2. One can see that $\tau$ diverges around $z \sim 1.8$ [15]. Such a value of the threshold parameter suggests that this transition might be related with the percolation transition in our network of interspecies interactions. To examine such a possibility we measured the average size of the largest cluster of connected links in the network $R$ (normalized by the number of species $N$ ) and the results are shown in Fig. 2 . Vanishing of this quantity locates the percolation transition [16]. One can see that the percolation transition takes place at a larger value namely around $z \sim$ 2.0. Our results suggest that these two transitions take place at different values of $z$. However the analysis of finite size effects especially in the estimation of $\tau$ is rather difficult and we cannot exclude that these two transitions actually overlap, as might be suggested by their proximity. Such a result would show that a dynamical regime of an ecosystem is determined by the geometrical structure of its interactions.

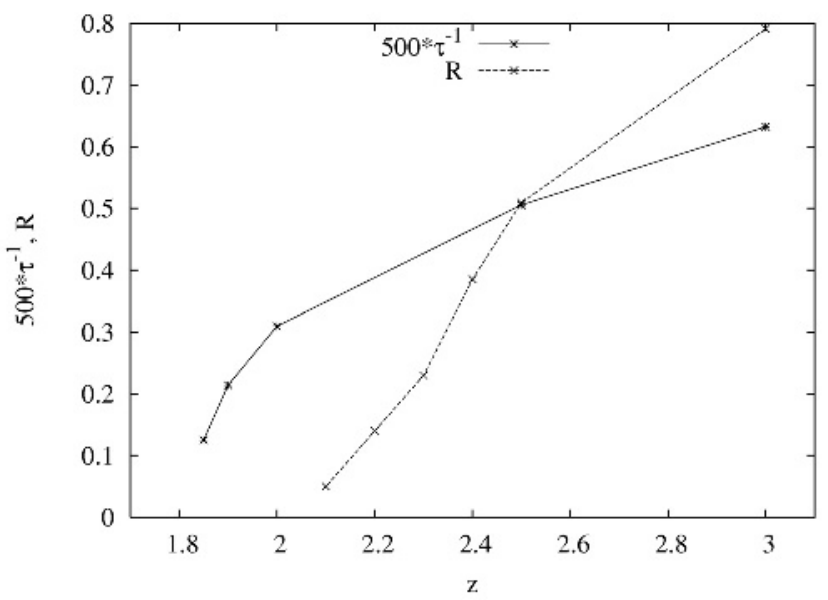

Fig. 2. The inverse average time between extinctions $\tau^{-1}$ and the percolation probability $R$ as a function of $z$. Plotted results are based on calculations for $N=100,200$, 300 and 400 and extrapolation $N \rightarrow \infty$

\subsection{Effect of a Modulated Growth Rate}

Now we examine the role of a modulated in time growth rate of preys. Such a modulation is supposed to mimic the influence of an external factor like a 
change of a climate. One of the questions that one can ask in this context is how such a change affects the extinction dynamics. We studied model I with $p(k)=1$. The growth rate of preys we chose as $g(t)=1+A \sin \left(\frac{2 \pi t}{T}\right)$, where $A$ and $T$ are parameters. A typical behavior in case of model I with such a growth rate is shown in Fig. 3. One can see that increased growth rate increases the

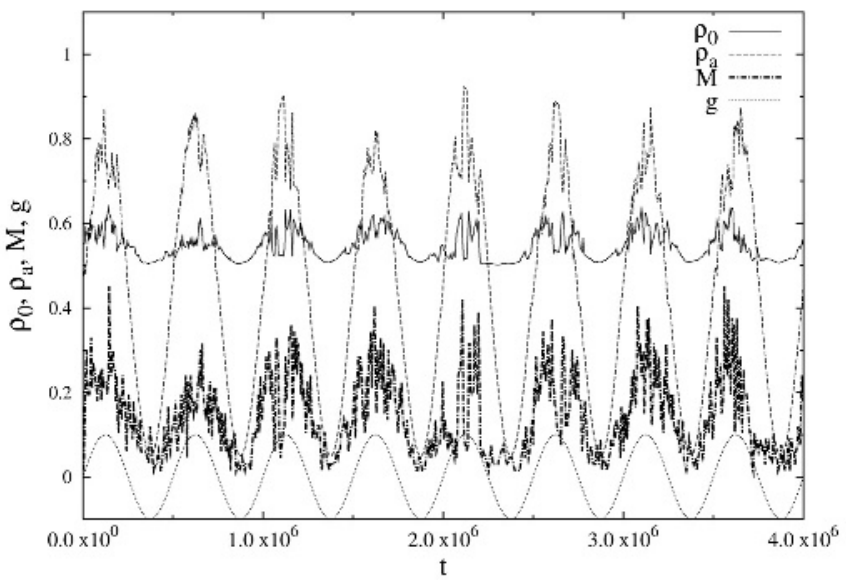

Fig. 3. A time evolution of the density of preys $\rho_{0}$, average density of predators $\rho_{a}=\frac{1}{N} \sum_{i=1}^{N}$, and the number of extinctions $M$ (divided by 20) in the time interval $\Delta t=10^{3}$ for the model I with $N=100$ and $z=4$. A rescaled modulated growth rate $(g(t)-1) / 10=0.09 \sin \left(\frac{2 \pi t}{T}\right)\left(T=10^{5}\right)$ is also shown

density of preys $\rho_{0}$ that increases the density of predators. However, it increases also the frequency of extinctions. Such a behavior, namely increased extinction rate during abundance of food, might at first sight look as counterintuitive. This effect is related with the form of environmental capacity terms in in the growth rate in Eq. 2, namely $1-\left(k_{i} \rho_{i}+\sum_{j}^{\prime} k_{j} \rho_{j}\right) /\left(k_{i}+\sum_{j}^{\prime} k_{j}\right)$. Such term certainly has a larger variability for increased density of predators $\rho_{i}$, and for some species (depending on the distribution of links, coefficients $k_{i}$ and densities) it causes faster extinction. Let us also notice that since period of modulation $T$ is quite large, there is no retardation effect between density of preys and predators. We observed such retardation for smaller values of $T(\sim 1000)$.

Modulated growth rate of prays affects also the probability distribution of intervals between extinctions $P(t)$ as shown in Fig. 4. One can see that period of modulation $T$ is imprinted in $P(t)$. Let us notice that certain paleontological data do show some signs of periodicity but its origin still remains unclear $[12$, 11].

It is known that slowly changing ecosystems sometimes undergo catastrophic shifts [17]. As a result, the ecosystem switches to a contrasting alternative stable state. It would be interesting to examine whether multi-species ecosystems, as 
described by our model (1)-(2), might also exist in such alternative states. If so, one can ask whether for example structure of the network of interspecies interactions or extinction dynamics are the same in such states.

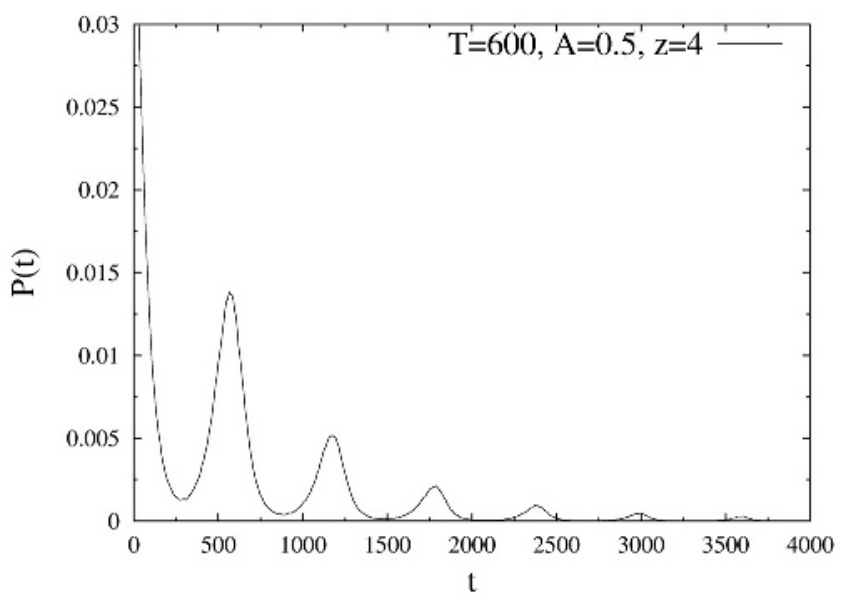

Fig. 4. Probability distribution of intervals between successive extinctions $P(t)$ calculated for model I with modulated growth rate $(N=100)$

\section{Conclusions}

In the present paper we studied extinction dynamics of a Lotka-Volterra model of a two-level food web. In our model $N$ species of predators feed on a single species of preys. Competition between predators, that is specified by a certain network of interactions, leads to their extinction and replacement by new species. Distribution of intervals between successive extinctions in some cases has powerlaw tails and thus resembles extinction pattern of the real ecosystem. However, when the network of interactions between predators is too sparse the ecosystem enters the steady state. We have shown that such a change of behavior might be related with a percolation transition of the network. We also examined an influence of external factors on the evolution of the ecosystem. More specifically, we studied the evolution of our model in case when the growth rate of preys is changing periodically in time. It turns out that such a modulation substantially changes the frequency of extinctions. Counterintuitively, periods with abundance of preys have higher frequency of extinctions than periods with lesser amount of preys.

It would be desirable to examine some extensions of our model. For example one can introduce additional trophic levels or other forms of interspecies interactions. One can also examine a variable number of species that would allow 
to create new species using certain mutation mechanism rather than assuming that they appear as soon as a niche becomes empty. Another possibility that is outside the scope of majority of macro-evolutionary models, and that will be discussed in the forthcoming paper [18], is to examine emergent properties of species.

Acknowledgement. This work was partially supported by the Swiss National Science Foundation.

\section{References}

1. J. D. Murray, Mathematical Biology, (Springer, 1989). J. Hofbauer and K. Sigmund The Theory of Evolution and Dynamical Systems, (Cambridge University Press, 1988).

2. P. Bak and K. Sneppen, Phys. Rev. Lett. 71, 4083 (1993). B. Drossel, Adv. Phys. 50, 209 (2001).

3. D. Chowdhury, D. Stauffer, and A. Kunvar, Phys. Rev. Lett. 90, 068101 (2003).

4. P. A. Rikvold and R. K. P. Zia, Phys. Rev. E 68, 031913 (2003).

5. M. Hall, K. Christensen, S. A. di Collobiano, and H. J. Jensen, Phys. Rev. E 66, 011904 (2002).

6. G. Abramson, Phys. Rev. E 55, 785 (1997).

7. B. Drossel and A. J. McKane, e-print: nlin.AO/0202034. D. Chowdhury and D. Stauffer, e-print: q-bio.PE/0311002.

8. C. Quince, P. G. Higgs, and A. J. McKane, in Biological Evolution and Statistical Physics, eds. M. Lässig and A. Vallerian (Springer Verlag, Berlin-Heidelberg 2002).

9. R. Albert and A. -L. Barabási, Rev. Mod. Phys. 74, 47 (2002).

10. One of the few macroevolutionary models that takes into account external factors was studied by Roberts and Newman (B. W. Roberts and M. E. J. Newman, J. Theor. Biol. 180, 39 (1996)). This model, however, is a variant of Bak-Sneppen model with dynamics operating at the level of species.

11. M. E. J. Newman and R. G. O. Palmer, e-print: adap-org/9908002.

12. D. M. Raup and J. J. Sepkoski, Proc. Natl. Acad. Sci. 81, 801 (1984).

13. Statistical properties of extinctions that we study in this paper are not affected by the precise value of the $\varepsilon$ as soon as it has a small value $(\varepsilon \ll 1)$.

14. The fact that we remove a species as soon as its density drops below $\varepsilon$ might suggest some similarity with Bak-Sneppen (BS) model. Let us notice, however, that in BS model at each time step a species is removed with the lowest fitness. In our model it is the dynamics that determines intervals between extinctions. Morevoer, in our model dynamical variables are densities of species, that in principle are measurable quantities, and not fitness.

15. For noninteger $z$ (i.e., the number of links of newly created species) we used the following procedure: with probability $z-[z]$ we created $[z]+1$ links and with probability $1+[z]-z$ we created $[z]$ links. On average such a recipe produces $z$ links.

16. D. Stauffer and A. Aharony, Introduction to Percolation Theory (Taylor \& Francis, London 1982).

17. M. Scheffer, S. Carpenter, J. A. Foley, C. Folks, and B. Walker, Nature 413, 591 (2001).

18. F. Coppex, M. Droz, and A. Lipowski, in preparation. 